\title{
May-Thurner syndrome: missed diagnosis and missed early treatment?
}

\author{
Alice Trinchero'; Sebastian Schotten²; Bernhard Lämmle'; Michael B. Pitton ${ }^{2}$ \\ ${ }^{1}$ Center for Thrombosis and Hemostasis (CTH), University Medical Center Mainz, Mainz, Germany; ${ }^{2}$ Institute for Diagnostic and Invasive Radiology, University Medical Center \\ Mainz, Mainz, Germany
}

A 24-year-old woman had suffered from left common iliac vein thrombosis at the age of 17 years, approximately 3 months after being started on a combined oral contraceptive pill, and she received INRadjusted phenprocoumon initiated under low-molecular-weight (LMW) heparin cover. The treating physicians detected heterozygous Factor V R506Q (Factor V Leiden) mutation and decided to extend phenprocoumon treatment up to one year.

Recurrent left iliac vein thrombosis occurred 6 years after incident deep vein thrombosis and approximately 4 weeks after left-sided hallux valgus surgery at the time of adequate perioperative LMWheparin prophylaxis discontinuation. She was treated with therapeutic-dose rivaroxaban for 1.5 years and addressed to the Center for Thrombosis and Hemostasis (CTH, Mainz) outpatient ward for advice on further anticoagulation.

She had moderately severe post-thrombotic syndrome (PTS) with discomfort and erythema of the left leg. May-Thurner syndrome was suspected and confirmed by magnetic resonance (MR) venography. $\checkmark$ panels a-c are showing severe compres-

Hämostaseologie 2017; 37: 184-185 https://doi.org/10.5482/HAMO-17-02-0009 received: February 5, 2017 accepted in revised form: April 20, 2017 epub ahead of print: July 24, 2017 sion and stenosis of the left common iliac vein (arrows), intraluminal web formation (asterisk) and post-thrombotic strictures in the common and external iliac veins (arrowheads). The patient agreed to catheter-directed dilatation and stenting ( panels $\mathrm{d}-\mathrm{i}$ ), which was performed to ameliorate symptoms of PTS. Right iliac venography ( panel d) was normal, whereas the left common iliac vein was compressed ( $\downarrow$ panel e, arrow) and showed web formation ( $\$$ panel e, asterisk). Balloon dilatation ( $\triangleright$ panel f) and placement of a dedicated venous stent ( $>$ panels $g, h$ ) with an increased radial force and an oblique design for the confluence of the inferior caval vein resulted in full patency of the left iliac vein axis ( panel i). Six months after stenting ultrasound examination showed full patency of the iliac veins ( $\downarrow$ panel j). The patient will be advised to discontinue rivaroxaban after 12 months of anticoagulant treatment from endovascular stenting.

May-Thurner syndrome, or iliac vein compression syndrome (1), is a common anatomic variation characterised by a compression and pulsatile damage of the left common iliac vein by the overlying right common iliac artery ( $\$$ panel $\mathrm{k}$, schematic anatomical picture), resulting in

- local venous endothelial damage,

- local intimal hyperplasia, and

- subsequent formation of intraluminal webs, bands or spurs (2).
The resulting venous stasis predisposes to left lower extremity and iliac vein thrombosis. Endovascular stenting may be indicated in addition to long-term anticoagulation in left common iliac vein thrombosis associated with the May-Thurner anomaly. An excellent long-term outcome after stenting with a stent patency up to $90 \%$ at one year or beyond has been demonstrated and stopping anticoagulant therapy after 1 year may be possible without significant risks of recurrent thrombosis or development of PTS (2).

May-Thurner syndrome should be mainly suspected in otherwise healthy women with pregnancy- or contraceptive pillassociated left iliac vein thrombosis (3).

\section{Conflict of interest}

The authors declare that there are no conflicts of interest.

\section{References}

1. May R, Thurner J. The cause of the predominantly sinistral occurrence of thrombosis of the pelvic veins. Angiology 1957; 8(5): 419-427.

2. Birn J, Vedantham S. May-Thurner syndrome and other obstructive iliac vein lesions: meaning, myth, and mystery. Vasc Med 2015; 20(1): 74-83.

3. Streiff MB, Agnelli G, Connors JM et al. Guidance for the treatment of deep vein thrombosis and pulmonary embolism. J Thromb Thrombolysis 2016; 41(1): 32-67. 


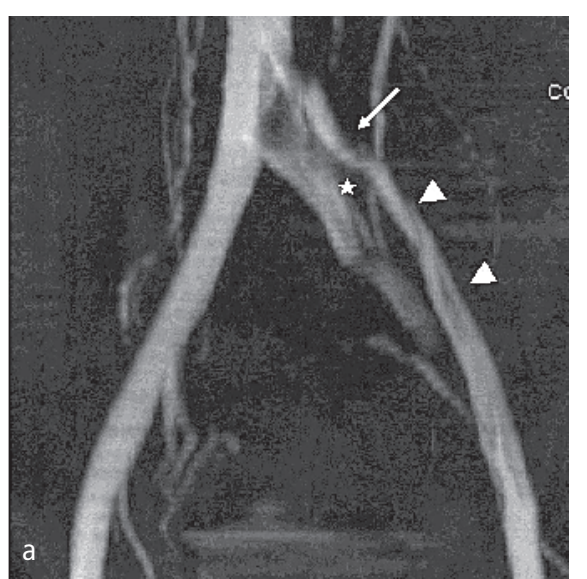

Fig. May-Thurner syndrome: missed diagnosis and missed early treatment?
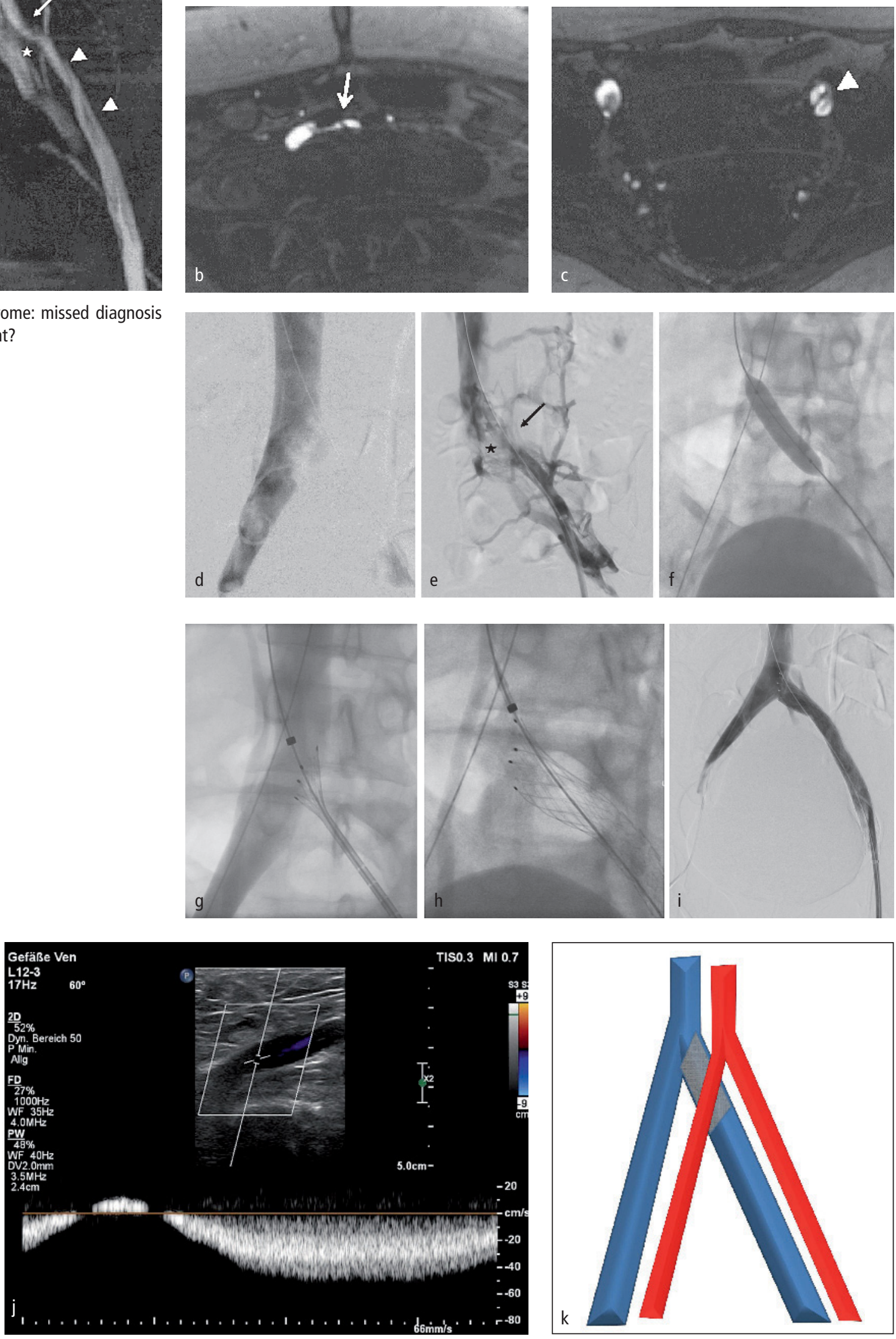